\title{
SHOCK WAVES AND PLASMA EJECTION: CORPUSCULAR AND INTERPLANETARY EVIDENCE
}

\author{
A. J. HUNDHAUSEN \\ High Altitude Observatory, National Center for Atmospheric Research*, Boulder, Colo., U.S.A.
}

(Presented by G. Newkirk)

\begin{abstract}
The ejection of rapidly-moving solar material into interplanetary space in association with solar flares has been discussed since 1859 , when geomagnetic disturbances and auroral displays followed shortly after the first observation of a flare by Carrington and Hodgson. Until the advent of in situ interplanetary observations in the early 1960's, such discussions were based upon the indirect information regarding interplanetary space that could be inferred from geomagnetic or cosmic ray data. The past decade of space exploration has provided a great deal of direct information regarding the interplanetary effects of solar flares and some quantitative implications regarding the nature of transient coronal disturbances.

This empirical information and related theoretical models have been reviewed by this author in several recent publications (Hundhausen, 1972a, b), to which the reader is referred for extended discussions. The most important implications of this work in the present context can be summarized as follows:

(1) Shock waves are observed to propagate past the orbit of the Earth, moving away from the Sun, after some solar flares. The typical shock wave sweeps past a stationary observer at $\sim 500 \mathrm{~km} \mathrm{~s}^{-1}$, and is thus moving at $\sim 100 \mathrm{~km} \mathrm{~s}^{-1}$ relative to the ambient solar wind.

(2) Shock wave observations reported in the literature imply an average rate of occurrence of several shocks per 27-day solar rotation. This rate of occurrence does not change drastically over the solar cycle.

(3) The rate given above directly implies that most flares do not produce shock waves that have been detected near the orbit of the Earth. Although it remains far from clear why some flares produce such disturbances while other flares, though at times of greater optical importance, do not, there is some comfort in the high correlation of interplanetary shock waves with the occurrence of related (i.e., both) type II and type IV radio bursts, the probable signature of plasma ejection and shock propagation in the corona.
\end{abstract}

(4) Integration of the excess (relative to ambient conditions) mass and energy fluxes in interplanetary shock waves gives the following estimates of the average mass $M$ and energy $W$ (at one solar radius) added to the solar wind

$$
\begin{aligned}
& M \approx 5 \times 10^{16} \mathrm{gm} \\
& W \approx 2 \times 10^{32} \mathrm{erg} .
\end{aligned}
$$

* The National Center for Atmospheric Research is sponsored by the National Science Foundation. 
The former is an appreciable fraction of a coronal mass, while the latter is as large or larger than any other known energy release in a large solar flare.

(5) The implication of mass ejection in association with interplanetary shock waves is consistent with the observation of post-shock solar wind with highly abnormal chemical (high helium content) and thermodynamic (low temperature) characteristics.

(6) Comparison of the observed post-shock variations in solar wind density and speed with theoretical models suggests that the mass and energy release occurs on a time scale of many hours. The lack of observational evidence for large mass motions in the corona associated with most solar flares, coupled with these long time scales, suggests to this author that the release of mass and energy into the solar wind is more extensive than that in the primary flare process. Perhaps the flare 'opens' a previously closed magnetic region, leading to emission of solar wind from a previously untapped coronal source region.

\section{References}

Hundhausen, A. J.: 1972a, in C. P. Sonnet, P. J. Coleman, and J. M. Wilcox (eds.), Solar Wind, NASA SP-308, Washington, pp. 393-421.

Hundhausen, A. J.: 1972b, Coronal Expansion and Solar Wind, Springer-Verlag, New York.

\section{DISCUSSION}

Brown: Some comments on the mass and energy figures you mention - first, though there are some uncertainties I will discuss later, it is not true that the $10^{32}$ erg in the blast greatly exceeds the energetic particle energy. Recent X-ray data indicate an electron energy entirely comparable with the blast energy.

If this is so and one assumes that the electrons are responsible for heating the thermal flare, then one correctly predicts a mass of around $10^{16} \mathrm{gm}$ in the high temperature flare, possible escaping. Also the fact that the $10^{32} \mathrm{erg}$ in the blast compares closely with the energy in the thermal flare is then not at all surprising on simple equipartition arguments.

Wild: The duration of the shock in interplanetary space suggest something similar to that of the radio storm continuum. Does anyone know if there is any correlation?

Newkirk: I am not sure anyone has looked into this.

Athay: Is it clear that what is attributed to the duration of the input is really that and not simply a dispersion in the paths followed by particles or some similar effect? In other words, is the conclusion that the input lasts for several hours the only possible one or could one construct reasonable alternatives?

Newkirk: But such dispersions would not modify the energy and mass estimates.

Sturrock: The blast wave may be the bow shock of a narrow plasmoid. If so, a 5 to $10 \mathrm{~h}$ duration for the piston is not required and the required mass would also be smaller.

Newkirk: If so, can you explain the observed broad angle covered by the shock? Also, unless the plasmoid were propelled to IAU, the narrow shock present close to the Sun would soon become nearly spherical.

Sturrock: This would depend upon the nature of the plasmoid. A great variety of shapes are observed in non-solar radio sources.

Dryer: The text makes the statement that additional theoretical consideration including the field are not needed at this time. I consider the incorporation of the magnetic field as an essential feature necessary to consider the ionized aspects of the interplanetary disturbances (including field compressions at the piston and anomalous transport properties - such as resistivity - indicated by theories presently in existence (such as Lee and Chen's) and presently being extended.

Newkirk: Do you refer to the effect of the field on the shock-disturbed flow or the effect of the field on the shock itself?

Dryer: The effect of the field on the disturbed flow.

Stewart: You did not discuss energy loss in the shocks. If white light observations near the Sun give sufficient energy, there is no energy loss. 
Newkirk: The comparison on an event by event basis is difficult since coronal events at the limb seldom reach the Earth. The calculations already include the gravitational energy loss.

Schmidt: The determination of the duration of the initiating disturbance from a fit of a model to the observed flow-profiles may not be so bad. In a diagram published by Hundhausen et al. indicating total energy vs total mass content of the disturbance, the observed shocks fall on a straight line of almost constant energy per mass. In this diagram the driven shock with increasing mass flow behind the shock fall nicely into the upper end of the diagram, i.e. they have larger mass - and energy content. So they should be caused by an initiating disturbance of longer duration. The shock waves with a blast wave profile fall into the lower part of the diagram and the intermediate profiles into the middle.

Zirin: Regarding the energy in different forms in a flare, Ramaty found for protons above $5 \mathrm{MeV}$ about $2 \times 10^{30} \mathrm{erg}$ for the 7 August 1972 event. Tanaka and Zirin found $2 \times 10^{30} \mathrm{erg}$ in $\mathrm{H} \alpha$ for the same flare.

Lin: The estimate (of $5 \times 10^{30} \mathrm{erg}$ ) by Ramaty of energy in $>5 \mathrm{MeV}$ protons in the August 4 flare is a gross underestimate of the total energetic particle energy since (1) the spectrum extends well below $1 \mathrm{MeV}$, and (2) the low energy protons lose some, perhaps most, of their energy in adiabatic deceleration in propagation outward in the interplanetary medium. These two effects can easily increase the energy content in energetic particles by orders of magnitude.

Brandt: The importance of the non-spherical geometry particularly near the sun can be illustrated with a simple example. Increasing the coronal temperature in the spherically symmetric case does not always produce an enhanced solar wind. If the coronal temperature exceeds about $4 \times 10^{6} \mathrm{~K}$, the gravitational nozzle vanishes, and no supersonic expansion can occur. For such high temperature regimes, the magnetic field may be needed to produce the nozzle which is necessary for a supersonic solar wind.

Brown (to Zirin): You quote $5 \times 10^{30} \mathrm{erg}$ in a flare but the point is that most of the electromagnetic radiation is in the EUV range and most of the thermal energy in the soft X-ray source. I don't know if data are available in these bands for the event you mention. 